\title{
A lunar clock changes shielding pigment transparency in larval ocelli
}

Gerta Fleissner ${ }^{\circ}$, Kirsten Schuchardt ${ }^{\circ}$, Dietrich Neumann*, Geetha Bali^^, Gerald Falkenberg $^{\#}$ and Guenther Fleissner ${ }^{\circ}$

○ Institut Zellbiologie \& Neurowissenschaften, J. W. Goethe-Universität Frankfurt a. M., Germany

* Zoologisches Institut, Universität Köln, Germany

$\wedge$ Center Biotechnology, Bangalore University, India

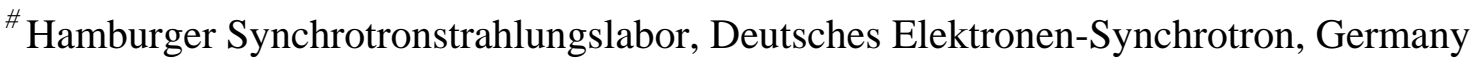

\section{Address correspondence}

Dr. Gerta Fleissner

Institute for Cell Biology and Neurosciences

Dept Biosciences

J.W.Goethe-University Frankfurt

Siesmayerstr. 70

D-60054 Frankfurt a. M., Germany

Tel +49-(0)69-798-24782

Email: fleissner@bio.uni-frankfurt.de 


\begin{abstract}
Living in the tidal zones of the sea requires synchronization with the dominant environmental influences of tidal, solar and lunar periodicity. Endogenous clocks anticipate those geoclimatic changes and control the respective rhythms of vital functions. But the underlying mechanisms are only partly understood. While the circadian clocks in animals are investigated employing neurobiological, molecular and genetic approaches, clocks with a lunar periodicity have been studied with reference to development and behavior only. Sites of their pacemakers, zeitgeber receptors, and coupled endocrine components are unknown. Here, a lunar-rhythmic change of shielding pigment transparency in the larval ocelli of the intertidal midge Clunio marinus is demonstrated for the first time as a possible access to the neurobiology of lunar timing mechanisms. We studied third instar larvae (Vigo strain) throughout the lunar cycle by light- and electron-microscopy as well as by x-ray fluorescence analysis for the identification of the pigment. Moonlight detection is a prerequisite for photic synchronization of the lunar clock. The larval ocelli of Clunio putatively may function as moonlight receptors and are also controlled by the circalunar clock itself, hence being primary candidates for tracing input and output pathways of the lunar pacemaker. Additionally, the demonstration of a reversible optical change of shielding pigment transparency in Clunio is a novel finding, not reported so far in any other animal species, and reveals a mechanism to enhance photosensitivity under the condition of very dim light. It represents a remarkable change of a sense organ from an imaging device to a radiometer. Its restriction to the developmental stage susceptible to lunar timing elucidates a unique sensory strategy evolved at the level of sensory input. It also raises basic questions about the biochemistry of optically active pigments, like melanin, and their intracellular control. (Author correspondence: fleissner@bio.uni-frankfurt.de)
\end{abstract}

Keywords: Clunio marinus, Intertidal midge, Melanosomes, X-ray-fluorescence analysis, Zeitgeber receptor 


\section{INTRODUCTION}

The endogenous timing of vital functions is an essential feature of all living organisms. These programs, biological clocks, anticipate the periodic events of the environment. Even under constant conditions, they continue running on periodicities close to the daily, semilunar, lunar or seasonal changes. They are termed circadian, circasemilunar, circalunar, circatidal, and circannual clocks, respectively. According to the habitat, physiological state and ecological needs of an organism, various clocks can be tuned to a complex timing program. The underlying mechanisms are well understood in case of the circadian clocks even down to the cellular and molecular level (Dunlap et al., 2004). In case of noncircadian clocks, however, nothing is known about the sites of pacemaker, zeitgeber receptors or other timing components (Saunders, 2002). Mainly, rhythms of reproduction and the development of marine organisms from various phyla (e.g. in brown alga Dictyota; polychaetes Eunice (Palolo) and Platynereis; sand-beach isopod Excirolana; grunion fish Leuresthes: for review: see Neumann, 1981) have been investigated with respect to their correlation to moon phases, but no neurobiological mechanisms have been examined.

The intertidal $1 \mathrm{~h}$-midge, Clunio marinus, is the best investigated model for circalunar rhythmicity interlaced with circadian rhythms (Neumann, 1966, 1989, 1995). It must synchronize its short imaginal life with spring tide low waters (occurring at days around full or new moon) to guarantee the reproductive success, which depends on temporal concentration of partners and egg laying on exposed substrates of the lower mid-littoral zone on rocky sea shores. For that purpose, the start of metamorphosis of Clunio is triggered in the last larval instar (L IV) (Neumann \& Spindler, 1991) in the case of the Vigo stock (Atlantic coast of Spain) by a circalunar clock (free-running period about 30 days). It is phase-controlled by nocturnal moon light, which obviously can be discriminated from the bright diurnal sunlight. Moonlight sensitivity is gated by a circadian clock, which evaluates only light stimuli during the hours before and after midnight as the relevant timing cue (Neumann, 1985). Up to now, the “moonlight receptor” has been unknown.

Here, the reversible conversion is shown of the larval ocelli from an image-forming eye into a radiometer, which may detect the dynamics of nocturnal moonlight as Zeitgeber signal for the lunar clock and a synchronised start of metamorphosis. Pioneering data concerning the nature of the shielding pigment from biophysical studies supported by x-rayfluorescence analysis strongly implicate melanin. As the change of transparency of the 
shielding pigment occurs also without exposure to nocturnal moonlight, it must be controlled by an endogenous lunar clock. This phenomenon might provide an initial paradigm for analysing neuronal components of a circalunar clock system - the input pathways for Zeitgeber information between ocelli and pacemaker as well as the output pathways between pacemaker and the ocelli as effector system.

\section{MATERIAL AND METHODS}

\section{Animals}

About 100 specimens of the third larval instar of the marine midge Clunio marinus (genetic strain Vigo- -Spanish Atlantic coast) were investigated (see Figure 1B). The animal were reared in synchronized mass cultures in light- and temperature-controlled chambers of the Zoological Institute in Cologne University in a 14:10 h light/dark (L/D) regime with maximum light intensity of about $5000 \mu \mathrm{W} / \mathrm{cm}^{2}$ (fluorescence light bulb OSRAM $40 \mathrm{~W} / 25 \mathrm{~S})$, constant temperature $\left(19^{\circ} \mathrm{C} \pm 0.5^{\circ} \mathrm{C}\right)$, and seawater (constant $15 \%$ salinity).Simulated moonlight (four consecutive nights illuminated by a $0.1 \mu \mathrm{W} / \mathrm{cm}^{2}$ light bulbs every 30 days) was also given to trigger the start of metamorphosis (seen Figure 1A). The days of this 30-day lunar cycle according to this time schedule; that is, LCD = lunar cycle day, starting with LCD 1 for the day with the first moonlit night until LCD 30 for the last day with a dark night. In nature, the first eight days of the lunar cycle have nocturnal moonshine, but in the lab, four moonlit nights suffice to entrain the culture (Neumann, 1981).

Larval may vary between 6 and 15 weeks at $20^{\circ} \mathrm{C}$; in general, they are exposed to 30day-moonlight cycles. The complex light conditions control control a switching point for the lunar periodic start of metamorphosis in the early fourth instar (Neumann, 1995) in synchronized cultures at LCD15 to LCD 20. Emergence from the pupae then occurs 18 to 20 days later at a certain time of day. The age of larvae can be individually determined under a stereomicroscope by the size of the larvae and the size and colour of the head capsule. In particular, the late larval stage III (which we investigated here) can be clearly discriminated from the early larval stage IV by the formation and size of the imaginal discs in the meso-and metathorax, which appear as soon as metamorphosis has started (Neumann \& Spindler, 1991). 


\section{Histological procedures}

We selected larvae of the same developmental age (larval stage III) and at well-defined lunar cycle days according to the lunar month in the laboratory. The number of samples (35 animals each) fixed per day is given in Figure 1B. The preparation and fixation of the animals were performed at four different times of day (9:30, 12:30,18:30, and 23:30 h CET) either under conventional light condition (cold light lamp) or an infrared stereo microscope. After cold anaesthesia, the larvae (maximum body length $1.5 \mathrm{~mm}$ ) were dissected into at least three parts in distilled water, in order to allow free access of fixative and staining chemicals without any salt crystallisation from seawater. The tissue was fixed in 3\% glutaraldehyde in $0.1 \mathrm{M}$ phosphate buffer ( $\mathrm{pH}$ 7.4) plus 3.4\% saccharose for about 6 $\mathrm{h}$ at room temperature. After rinsing in the same buffer-saccharose mixture, part of the samples were postfixed in $2 \% \mathrm{OsO}_{4}$ ( in buffer) for about $1.5 \mathrm{~h}$. Samples for the $\mathrm{x}$-rayanalysis were not postfixed in $\mathrm{OsO}_{4}$. After another rinsing and dehydration, the tissue was resin-embedded (Araldite) and cut into 3-4 $\mu$ m-thick semithin sections. Control sections were stained with methylene blue (standard recipe).

Appropriate sections were selected for re-uptake, and cut into 40 to $60 \mathrm{~nm}$ ultrathin sections. The sections on grids were contrasted with $10 \%$ uranylacetate in methanol (10 min), rehydrated, stained with Reynold’s lead-citrate (7 min), and washed in distilled water with $0.01 \mathrm{~m} \mathrm{NaOH}$ (15 min) and twice in clear distilled water.

\section{X-ray fluorescence analysis}

As biochemical studies on single ocelli of Clunio are not possible due their size (diameter less than $20 \mu \mathrm{m}$ ) and fast-changing structure during larval development and with lunar phases, physicochemical methods were used to characterize the shielding pigment. $\mu$-X-ray-fluorescence-analysis ( $\mu$-XRFA) yields accurate qualitative and quantitative information on the element composition of a certain material without destroying its configuration (see Janssens et al., 2001). Test samples for $\mu$-XRFA were processed in a clean environment to prevent contamination. Tools and vessels were free of iron and other transitions metals. The midges were fixated in 3\% glutaraldehyde in $0.1 \mathrm{~m}$ phosphate buffer, embedded in Araldite, and cut into semithin sections ( $3 \mu \mathrm{m}$ thick) by a glass knife. This tissue was neither postfixed with $\mathrm{OsO}_{4}$ nor stained with any contrasting agents. In standard recipes for electron microscopic sample preparation, cacodylate buffer is 
recommended instead of the phosphate buffer used here. Phosphate buffer was used instead of cacodylate buffer, which contains arsenic acid and which would give an unspecific fluorescence signal in $\mu$-XRFA.

The semithin sections were mounted on Ultralene foil (SPEX CertiPrep 2003 Metuchen, NJ, USA), which has only few well-known peaks of autofluorescence. The measurements were performed at beam $\mathrm{L}$ at the Hamburger Synchrotron Laboratory (HASYLAB) at the Deutsche Electron Synchrotron facility (DESY). The x-ray capillary allowed a topographic element scan with an resolution of 10 - $15 \mu \mathrm{m}$. After calibration by means of reference material, the quantitative values of certain elements can be calculated and then drawn as topographic distribution of the respective mineral in the test sample. Semithin sections of the same unstained specimens were cut into ultrathin section for an electron microscopic analysis of the subcellular localisation of minerals.

\section{Documentation}

A Polyvar microscope (Reichert, Vienna, Austria) equipped with a high-resolution digital microscope camera (Spot; Diagnostic instruments, Sterling Heights, Ill., USA) and Metaview software (version 3.6; Universal Imaging Corporation, West Chester, Pa., USA) was used for the light microscopic inspection of the slides and the documentation of the results. We used the electron microscopes Zeiss C10R and C12R (Oberkochen, Germany). for inspection of the ultrathin sections. The published versions of the figures were processed by means of Adobe Photoshop software (version CS 8.0.1; Adobe systems, Mountain View, Calif., USA).

\section{RESULTS}

The translucent Clunio larvae are about $1.5 \mathrm{~mm}$ long and 200 to $300 \mu \mathrm{m}$ in diameter. They have two pairs of ocelli (see Figure 2) with a lens and a cup-shaped retina with Vshaped rhabdomeres forming a star-like rhabdom (see Figure 3). Unpigmented smaller ocelli (diameter less than $10 \mu \mathrm{m}$ ) are situated in front of larger ocelli (diameter about $20 \mu \mathrm{m}$ ), which are dark brown from shielding pigment granules inside the photoreceptor cells (see Figure 3). Separate pigment cells are missing. The pigmented ocellus - as an image processing device - has a directionality for receiving light only through a small angle of acceptance from one main direction. This is characteristic for this type of eye, which is 
shielded against light from the back. Further on, the unpigmented ocelli will not be addressed, as they may stem from vestigial stemmata of so far unknown biological meaning.

When the large ocelli of specimens from all lunar phases (LCD 1 - 30, see Figure1B) were investigated, the optical situation in the retina dramatically changed in moonlit nights: the normally "black-brown" shielding pigment granules (see Figures 3A, 3C; and 4B) then appeared completely transparent (see Figures 3B, 3D, and 4A), a phenomenon lasting for about eight days (LCD 1-8), a period similar to the natural timespan of nocturnal moonlight. The subcellular compartments of the pigment granules still were preserved (see Figures 4A, and 5A-5C). They consist of closely packed vesicles of smooth endoplasmic reticulum (see Figures 4A, 5B, and 5C). Cell motor organelles, like microtubules and microfilaments, are missing in this receptor cell area. After LCD 8-9, the refill of shielding pigment, fluffy electron-dense material, occurs gradually from the centre to the rim. The margin of the endoplasmic vesicles sometimes seem to be overloaded with electron-dense material with connection to the centrally located pigment (see Figures 5D, and 7J). Within 3 - 4 days, the granules turn back to their "normal black" light-absorbing state (see Figure 5E). They stay like this for about two weeks (see Figures 5D, and 5E). Shortly before the next nocturnal moonlight is expected (LCD 26-27), the black appearance slowly turns transparent. This process starts from the periphery of the granule (see Figures $4 \mathrm{~B}$, and $5 \mathrm{~F}$ ) and gives a starlike appearance of the pigment. This lunar cycling of shielding pigment transparency neither depends on the time of day nor on the light program the larvae have experienced prior to fixation. The changing pigment transparency is evident even when the artificial moonlight in the laboratory is omitted (see Figures 3C, and 3D).

In order to determine the chemical nature of the shielding pigment, a topographic highresolving element analysis was applied by means of $\mathrm{x}$-ray microspectroscopy ( $\mu$-XRFA: micro-X-ray-fluorescence-analysis).The element scans offer a clear interpretation (see Figure 6): The element spectrum of a pixel inside the ocellus (Figure 6A: high iron content) is clearly different from the spectrum of a site away from the ocellus in the head capsule (Figure 6B: higher calcium due to nervous tissue) and outside the capsule (Figure 6C). The XRF-mappings of the head capsule (see Figures 7A-7F) for iron, calcium, chloride, potassium, and zinc (not shown) match the site of the ocellus, while manganese can be found in the head capsule. At higher resolution, the ocellus resembles a tiny iron cup (Figure $7 \mathrm{H}$ ) containing a small area of high calcium (Figure 7I): iron co-localizes with the area of the ocellar shielding pigment, while the rhabdoms have higher calcium content. The specimens tested here stem from different lunar phases. The sample shown in Figures 7A-7F 
is fixed at the end of the natural nocturnal moonshine (LCD 8) and has transparent pigment as can be seen in the control section (see Figure7A). The specimen of Figures 7G-7I is fixed during new moon at LCD 22 and has dark brown pigment, but still the iron content inside the ocellus stays the same. Electron microscopy of the Clunio pigment granules in samples without $\mathrm{OsO}_{4}$ and contrasting agents (see Figure 7J) shows the putative site of the transition metals as irregularly packed "striped" electron opaque material concentrated next to the margin of the smooth endoplasmic reticulum.

\section{DISCUSSION}

The larval eyes of Clunio marinus change their appearance reversibly during the lunar cycle. Driven by a lunar clock, the shielding pigment “disappears” for about a week, when naturally the moon would shine at night, and reappears afterward. This phenomenon has not yet been described in any other organism. Two main questions arise from this finding:

- Which is the biochemical nature of the pigment?

- What is the biological meaning of this phenomenon?

\section{The Nature of This Pigment}

Basically, two types of retinal shielding pigments are found in arthropods, ommochromes, and melanins (Needham, 1974), which may occur in manifold variations. As the two classes of shielding pigments differ not only in the biochemistry but also in their content of transition metals, we applied high-resolving element analysis by the means of $\mathrm{x}$ ray microspectroscopy (Fleissner et al., 2007; Stahl et al., 2006). This approach of a topographic analysis of intact tissue samples is based on the element-specific fluorescence, which can be investigated by $\mu$-XRFA (micro-X-ray-fluorescence-analysis). The element scans offer a clear interpretation (see Figures 6 and 7). The retinal shielding pigment of Clunio contains transition metals, primarily iron, which constitute evidence for melanin rather than ommochrome. Another observation underlines the assumption that the ocellar pigment may be a melanin; in immunohistological procedures, the usual treatment of sections with $\mathrm{H}_{2} \mathrm{O}_{2}$, bleaches the pigment granules in Clunio, while the same procedure applied to, for example, beetle eyes had no effect on their shielding pigment granules (Fleissner et al., 1993). 
The molecular components of the shielding pigment most likely stay in place within their granules because the content of Fe and other heavier elements (e.g., Zinc) in the retina does not change in spite of the optical change from opaque to transparent (Stahl et al., 2006); however, they may change their molecular configuration in a way that alters their optical appearance. Another option would be the transport in and out through the SER-membrane, which shows the remarkable concentration of "dense" material, and an intragranular lattice, which is assumed to form a pathway for mineralization. Ongoing experiments with infrared microscopy will hopefully determine more details of the underlying processes, which may parallel those of melanization in different pigment cells (e.g., Eibl.et al., 2006). However, these mechanisms generally occur unidirectionally (i.e., as a filling of melanosomes). The reverse, depletion of melanin, as is observed in Clunio, is otherwise found as degenerative process, only; thus, the larval ocelli offer the unique opportunity to investigate the mechanisms of cycling melanization.

\section{The Putative Biological Meaning}

In various animals, rhythms of shielding pigment migration occur under exogenous (light-induced) or endogenous (clock-induced) control (see Fleissner \& Fleissner, 2006). In all these cases, the pigment is preserved, but moved to either occlude or expose the lightsensitive rhabdoms to match the incident light intensity. Only circadian, but no circalunar, clock-controlled phenomena have been reported. The "optical disappearance" of shielding pigment, as observed in the ocelli of Clunio, is a novel phenomenon.

What could be the functional significance of the optical neutralization of shielding pigment in the ocellus?

1. Basically, the transparency of shielding pigment enables the ocellus to catch more of the few available photons, which may reach the Clunio larvae in their shelter at the seafloor during moonlit nights. The directionality of the ocellus is mostly gone, when the pigment is transparent. But inside their self-molded tubes, within the intertidal substrate, they probably only require a simple light-dependent spatial orientation. Further information on the vision physiology of Clunio is lacking. Both aspects, together, match the properties of a sensitive radiometer that integrates incident light over space to thus measure the dynamics of moonlight.

2. Another aspect in favor of a function of these larval ocelli as a moonlight zeitgeber receptor is that their temporal transparency is gated corresponding to the natural period of nocturnal moonlight. This makes the system less sensitive during the rest of the month. 
The intensity peak of nocturnal moonlight after dusk and well before dawn contains a rather sharp timing signal within the lunar periodicity. In laboratory experiments (Neumann, 1985), it could be shown that nocturnal light, occurring in four consecutive nights, works as a zeitgeber for the circalunar clock. For this project, the specimens were collected from a laboratory stock with their larval development experimentally synchronized by the daily L/D cycle. The duration of the larval development may individually vary at $20^{\circ} \mathrm{C}$ between 6 and 15 weeks. Only one lunar zeitgeber exposure during the larval stage III would be sufficient to evoke metamorphosis and eclosion.

The discrimination between random and periodic events by the evaluation of their repetition within certain gates has been reported in various animals (Neumann, 1976). Probably the ocellar "moonlight window" is part of a complex photosensitive system that enables a precise gating and filtering of photic events in order to discriminate relevant periodic illumination from random light stimuli. The observation that nocturnal moonlight during four successive days of the entraining 30-day cycle is only effective as a synchronizing stimulus during the hours around "subjective midnight” and not during “subjective day, dawn, and dusk” suggests an additional circadian gate (Neumann, 1995).

The "moonlight window" of the larval ocelli of Clunio seems to be not only an appropriate input stage for Zeitgeber stimuli to the circalunar clock, but it also presents an output system that receives efferent controlling signals from the lunar pacemaker. Thus, it seems feasible to localize the pacemaker site by either anterograde or retrograde tracing of the signalling pathways. Pilot studies of the distribution of biogenic amines in Clunio larvae already delivered promising results (Fleissner and Schuchardt, unpublished). The retina positively reacts with anti-5HT-antibodies, suggesting a serotonergic efferent control. Small compartments in the cross-section of the optic nerve contain neurosecretory granules. Another first hint towards a fiber-bound efferent control can be derived from the observation that the darkening of the shielding pigment always starts near the postretinal membrane, where the optic nerve leaves the eye. Further genetic and fine structural investigations, also with regard to the circadian modulation of the moonlight perception via extraretinal photoreceptors, are in progress to elucidate the neuronal interaction of circadian and circalunar timing systems of Clunio. 


\section{ACKNOWLEDGMENTS}

This work is part of a project supported by grants from the Deutsche Forschungsgemeinschaft to G. F. (Fl 177/10-1,2) and to D.N.(Ne 72/32-1,2), a HumboldtFellowship to G.B. (INI 1001332), and project grants of the HASYLAB at DESY (I-04-012, I-05-94). The authors gratefully acknowledge the support of B. Stahl (TU Darmstadt) during pilot tests at the Synchrotron Lab Hamburg (HASYLAB) and the discussions on the physicochemical nature of the shielding pigment with G. Weseloh (U Potsdam). C. TaylorDorenkamp (U. Boston) helped to revise the English version of this manuscript. The experiments were performed in agreement with the rules and regulations on animal welfare in Germany and Chronobiology International (Touitou et al., 2006). The general regulations, policies, and principles given in the "Guide for the Care and Use of Laboratory Animals” were followed.

\section{REFERENCES}

Dunlap JC, Loros JJ, DeCoursey PJ (eds). (2004). Chronobiology: Biological Timekeeping. Sinauer Associates, Sunderland, MA., 406 pp.

Eibl O, Schultheiss S, Blitgen-Heinecke P, Schraermeyer U. (2006). Quantitative chemical analysis of ocular melanosomes in the TEM. Micron 37: 262-276.

Fleissner Ge, Fleissner Gu. (2006). Endogenous control of visual adaptation in invertebrates. In Warrant EJ, Nilsson DE (eds.). Invertebrate vision. Cambridge: Cambridge University Press, pp. 127-166.

Fleissner Ge, Schuchardt K, Stahl B, Neumann D, Fleissner Gu. (2007). Melanosomes in a larval ocellus of an intertidal midge. DESY Annual Report 2006, pp. 1337.

Janssens KHA, Adams FCV, Rindby A. (eds.). (2001). Microscopic X-ray fluorescence analysis. Chichester-New York: Wiley, 419 pp.

Needham AE. (1974). The Significance of Zoochromes. Berlin, Heidelberg, New York: Springer, $429 \mathrm{pp}$.

Neumann D. (1966). Die lunare und die tägliche Schlüpfperiodik der Mücke Clunio. Steuerung und Abstimmung auf die Gezeitenperiodik. Z. vergl. Physiol. 53:1-61. 
Neumann D. (1976). Entrainment of a semilunar rhythm. In DeCoursey PJ (ed.) Biological rhythms in the marine environment. Columbia, S.C. (USA): University South Carolina Press, pp. 115-127.

Neumann D. (1981). Tidal and lunar rhythms. In Aschoff J (ed.) Handbook of Behavioral Neurobiology. New York: New York Press, pp. 351-380.

Neumann D. (1985). Photoperiodic influences of the moon on behavioral and developmental performances of organisms. Int. J. Biometeorology 29:165-177.

Neumann D. (1989). Circadian components of semilunar and lunar timing mechanisms. In Daan S, Gwinner E (eds) Biological clocks and environmental time. New York: The Guildford Press, pp. 173-182.

Neumann D. (1995). Physiologische Uhren von Insekten. Naturwissenschaften 82:310-320.

Neumann D, Spindler KD. (1991). Circasemilunar control of imaginal disc development in Clunio marinus: temporal switching point, temperature-compensated developmental time and ecdysteroid profile. J. Insect Physiol. 37:101-109.

Saunders DS. (2002). Insect clocks, 3rd ed. Amsterdam: Elsevier Science, 560 pp.

Stahl B, Fleissner Ge, Fleissner Gu, Schuchardt K, Neumann D. (2006). Element scanning of shielding pigment in the larval ocellus of Clunio marinus.. DESY annual report 2005, pp. 1031-1032.

Touitou Y, Smolensky MH, Portaluppi F. (2006). Ethics, standards, and procedures in human and animal research in chronobiology. Chronobiol. Int. 23:1083-1096. 
Figures

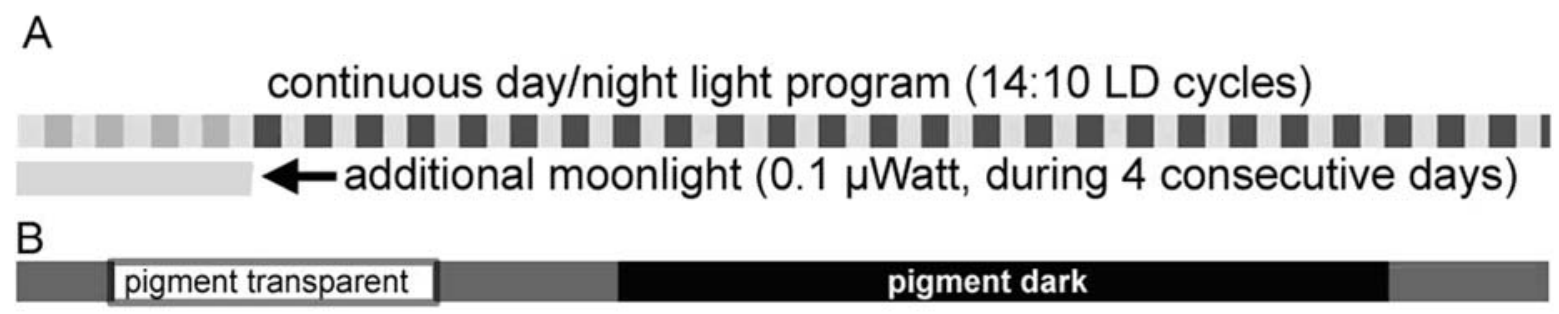

FIGURE 1 (A) Light program for the synchronization of metamorphosis with solar and lunar periodicities in the laboratory. Mass cultures of Clunio are reared under 14:10 h L:D cycles. On four consecutive days, an artificial nocturnal light simulates nocturnal moonshine. In nature, this period would last about a week. (B) Number of samples investigated at different days of the lunar cycle. The numbers shown here include 3 - 5 animals each, which were processed simultaneously. Numbers with an asterisk indicate that some of these samples have not seen nocturnal moonlight.

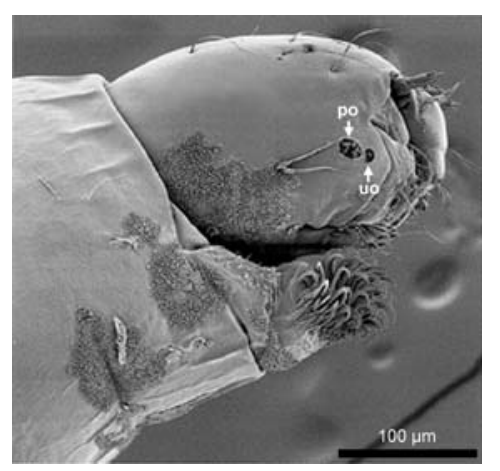

FIGURE 2 Scanning electron microscopic lateral view of a Clunio head. As the ocelli are not visible from the outside, we depicted their position. Abbreviations: po = pigmented ocellus, uo = unpigmented ocellus. 


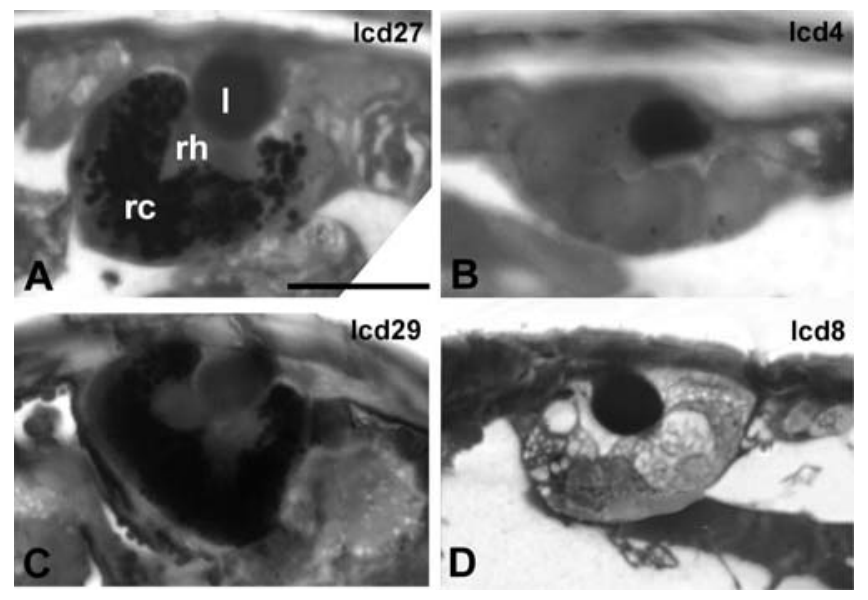

FIGURE 3 Larval ocellus of Clunio marinus in different lunar phases. Light microscopic view of semithin (4 $\mu \mathrm{m}$ thick Araldite) sections fixed at different lunar cycle days: A: LCD 27, B: LCD 4, C: LCD 29, and D: LCD 8. During the period of nocturnal moonlight (B and D), the ocelli loose their dark-brown pigmentation, which otherwise can be seen clearly (A and C). Although the larvae fixed for $\mathrm{C}$ and $\mathrm{D}$ did not receive simulated moonlight, the pigment cycling persists and thus must be endogenously controlled. (Scale bar $30 \mu \mathrm{m} ; \mathrm{l}$ = lens, rc = receptor cell, $\mathrm{rh}=$ rhabdom).

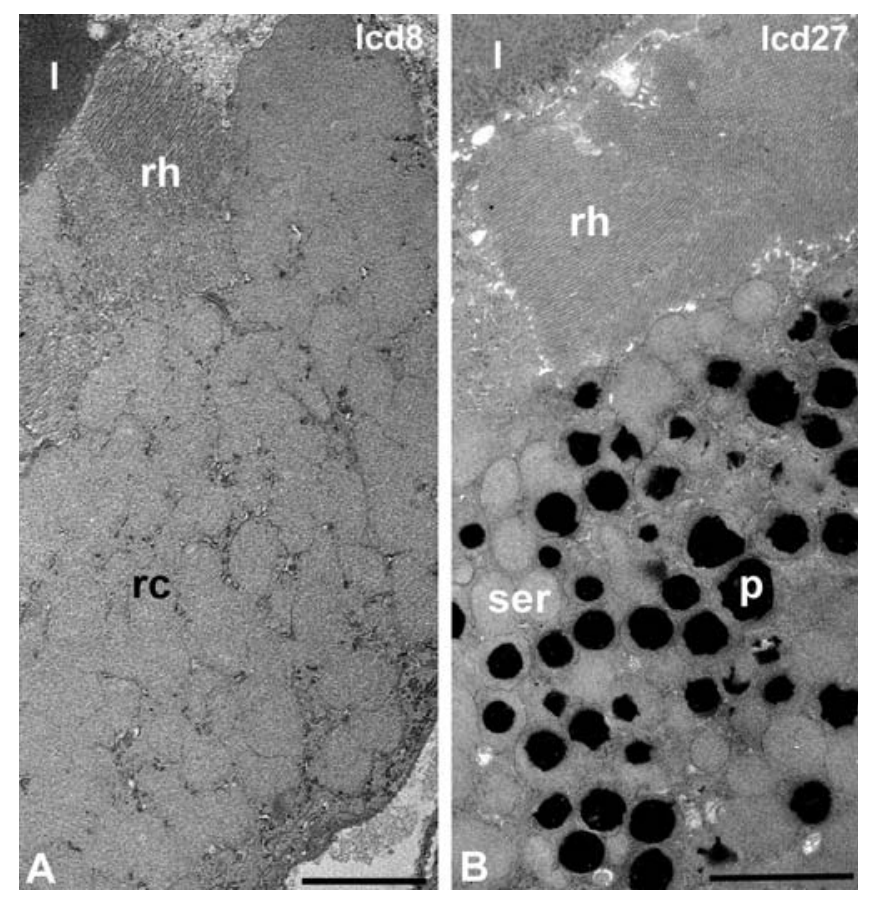

FIGURE 4 Electron microscopic section through an ocellus during full moon (A: fixed at LCD 8) and new moon phase (B: fixed at LCD 27). The vesicles of smooth endoplasmic reticulum are "empty" during moonlit nights (A) and filled with pigment in the lunar phase without nocturnal moonshine (B). (Scale bar $5 \mu \mathrm{m}$; l = lens, rh = rhabdom, $\mathrm{p}=$ shielding pigment, $\mathrm{rc}=$ receptor cell, ser $=$ smooth endoplasmic reticulum). 


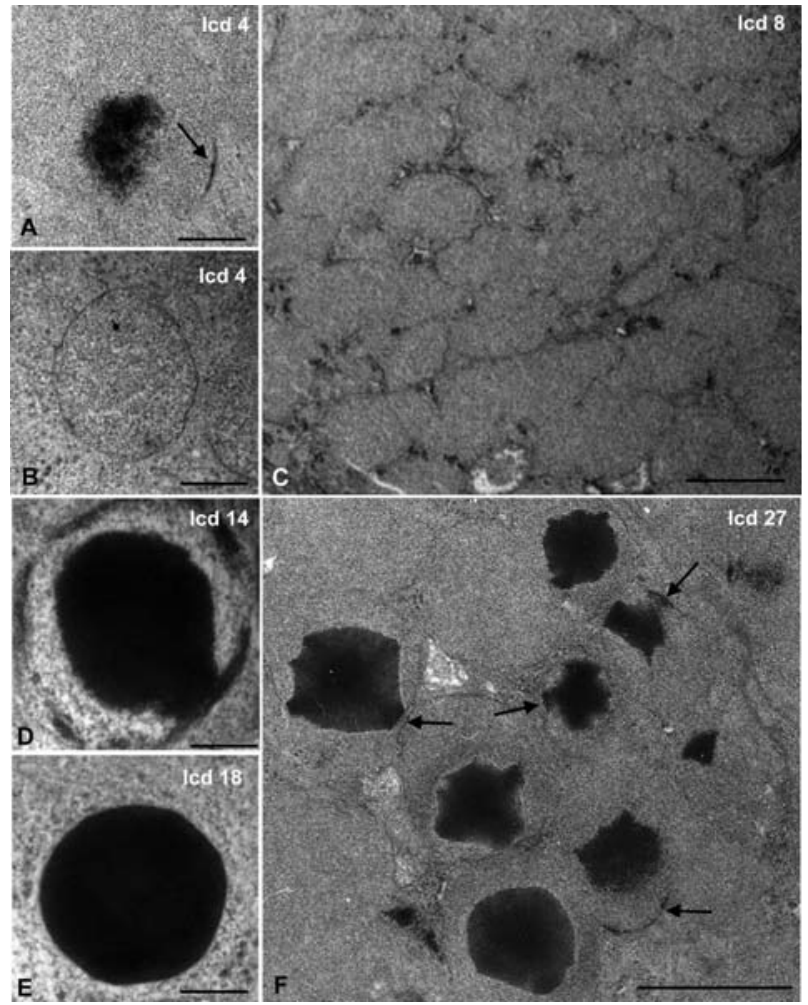

FIGURE 5 Shielding pigment turnover during different lunar phases. At LCD 4, nearly all shielding pigment is gone (A and B). Few granules show residues of pigment and blackened membrane areas (arrow in A), and most vesicles are empty. (C) In LCD 8, not a single pigment granule can be found. (D, LCD 14) Afterward, the granules slowly refill (pigment "arising" from a thickened membrane), and (E, LCD 18) all are black for about 14 days. (F, LCD 27) Already 3 to 4 days before nocturnal moonlight, the pigment seems to retract from the granular membrane (arrows) in a star-like appearance. (Scale in A, B, D, E, $0.2 \mu \mathrm{m}$; scale in C and D, $200 \mu \mathrm{m}$ ).

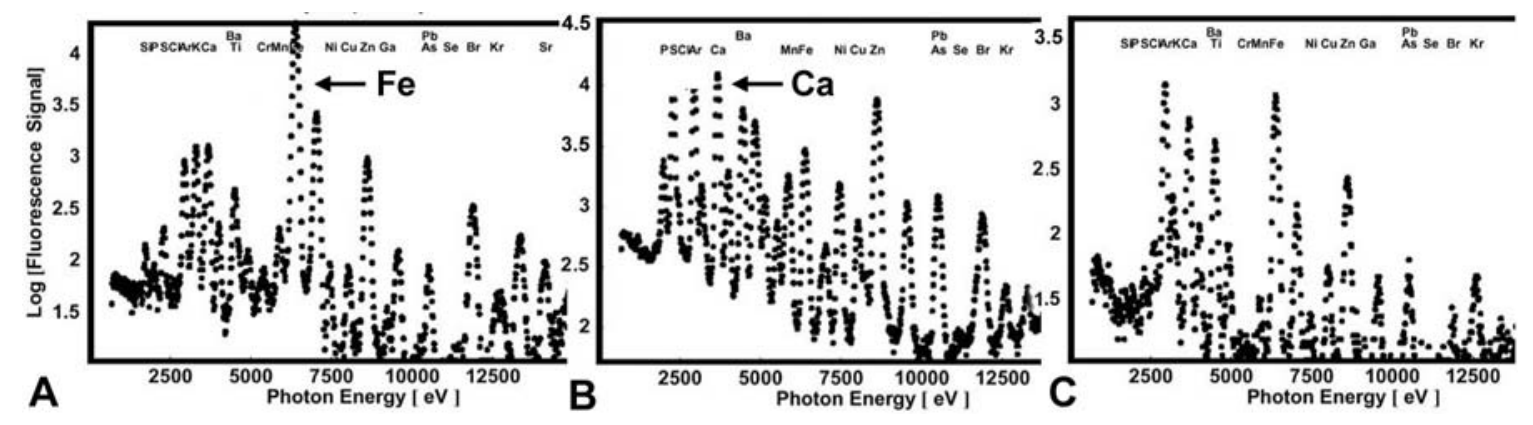

FIGURE 6 High-resolution analysis of element distribution by $\mu$-X-ray fluorescence analysis ( $\mu$ XRFA) performed at Beamline L at HASYLAB (DESY, Hamburg). The analysis of the intact tissue gives different fluorescence spectra, indicative of the topographic element composition (A) within the ocellus (high iron concentration), (B) outside the ocellus but inside the head capsule (lower iron but still high calcium concentration) and (C) outside the head capsule. Note: y-axis normalized. 

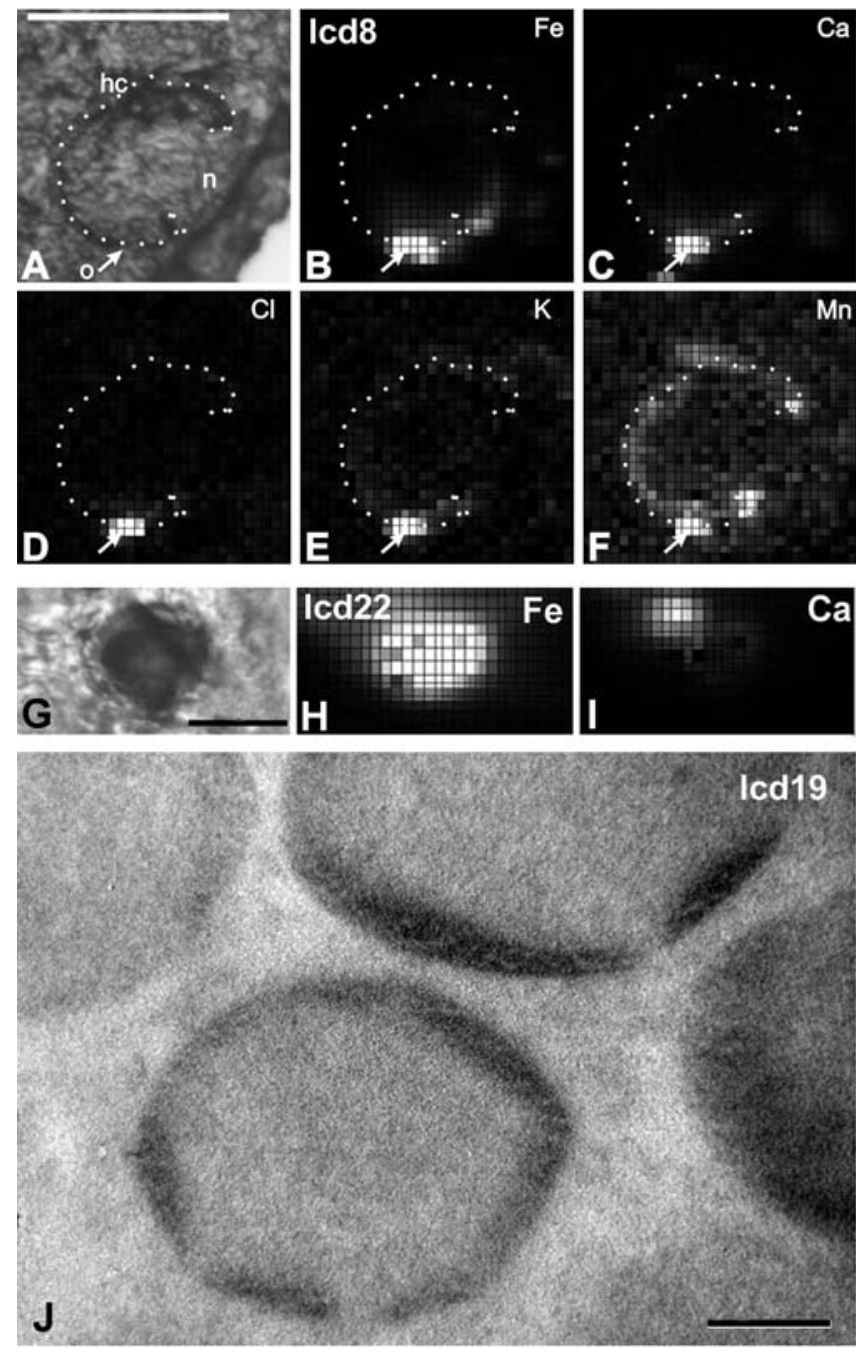

FIGURE 7 Topographic $\mu$-x-ray fluorescence mapping of a head capsule $(A-F)$ and of an ocellus (G-I). A and G show the control light microscopic views of the test samples mounted on Ultralene foil. A t F: The head capsule (hc) is stippled; the site of the ocellus (o) is shown by an arrow in A - F ( $n$ = neck; animal fixed at LCD 8). The ocellus has a high concentration of iron (Fe), calcium (Ca), chloride (Cl) and potassium (K); the head capsule contains different minerals, such as manganese (Mn). (Scale bar $200 \mu \mathrm{m}) . \mathrm{H}$ - I: At a higher resolution, x-ray spectroscopy shows iron (Fe) at the site of the shielding pigment, and calcium ( $\mathrm{Ca}$ ) indicates the position of the rhabdom of the ocellus. (Scale bar $20 \mu \mathrm{m}$; animals fixed at LCD 22). J: In non-osmicated, ultrathin sections, the pigment granules have an electron-dense margin indicating the localization of these transition metals. (Scale bar $0.2 \mu \mathrm{m})$. 